\title{
Thyroid sonography as an extension of the bedside examination in hyperthyroidism
}

\section{Angelos Kyriacou}

CEDM Centre of Endocrinology, Diabetes \& Metabolism, Limassol, Cyprus; Diabetes, Endocrinology \& Obesity Medicine, Salford Royal NHS Foundation Trust, Salford, Greater Manchester, UK

(iD) https://orcid.org/0000-0003-2282-0192

Corresponding author: angelos5@doctors.org.uk

\section{Panayiotis A Economides}

School of Medicine, European University Cyprus, Nicosia, Cyprus

(iD) https://orcid.org/0000-0002-5504-5113

\section{Akheel A Syed}

Division of Diabetes, Endocrinology \& Gastroenterology, Faculty of Biology, Medicine \& Health, The University of Manchester, Manchester, UK

(D) https://orcid.org/0000-0001-8696-7121
DOI: https://doi.org/10.20883/medical.e482

Keywords: hyperthyroidism, graves, sonography, ultrasound, point-of-care

Published: 2020-12-29

How to cite: Kyriacou A, Economides PA, Syed AA. Thyroid sonography as an extension of the bedside examination in hyperthyroidism. JMS [Internet]. 2020 Dec 29;89(4):e482. doi:10.20883/medical.e482

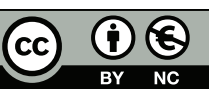

(C) 2020 by the author(s). This is an open access article distributed under the terms and conditions of the Creative Commons Attribution (CC BY-NC) licencse. Published by Poznan University of Medical Sciences

\begin{abstract}
In this mini-review, we discuss the role of thyroid sonography as a 'point-of-care' tool for assessing and managing patients with (suspected) hyperthyroidism who present to the endocrine outpatient clinic. A thyroid ultrasound may aid in distinguishing between hyperthyroidism and destructive thyroiditis. The presence of intense vascularity ('thyroid inferno') on the power Doppler has a very high positive predictive value in identifying hyperthyroidism. It may also allow for the sub-classification of hyperthyroidism into autoimmune and nodular hyperthyroidism. It is important to identify the presence of thyroid nodules at an early stage, as this may influence management. Toxic nodules requires definitive treatment, as well as the presence of nodules in Graves' disease because of increased risk of malignancy. Current guidelines on hyperthyroidism do not clearly state thyroid sonography as a first line investigation, although recent authoritative reviews point in that direction. Given the aforementioned benefits of thyroid sonography, alongside the reduced costs and widespread availability of high-resolution (including portable) ultrasound devices, there is an argument for thyroid sonography to be applied as a first line investigation for all patients with hyperthyroidism. Endocrinologists trained in thyroid sonography could perform this as an extension of their clinical examination when patients first present with hyperthyroidism at the endocrine clinic.
\end{abstract}

Thyroid ultrasound scanning (USS) is the most sensitive imaging modality for assessing the morphology of the thyroid. It is also the most commonly accepted imaging modality for assessing thyroid nodules, including its application in guiding fine needle aspirations, and in the long-term monitoring of patients who were treated for thyroid cancer (alongside tumour markers).
However, thyroid USS is infrequently utilised in the investigation of patients presenting primarily with thyrotoxicosis. The differential diagnosis of thyrotoxicosis is wide [1], and includes conditions that cause increased thyroid hormone production and secretion (hyperthyroidism; e.g. Graves' disease (GD), toxic multi-nodular goitre and solitary toxic nodule) and conditions that do not (e.g. 
thyroiditis and factitious thyrotoxicosis). Thyroid USS can often help differentiate hyperthyroidism from destructive thyroiditis and other thyrotoxicosis-causing conditions, such as factitious thyrotoxicosis and struma ovarii, which are associated with a morphologically-normal thyroid gland. Similarly, thyroid USS can promptly differentiate between autoimmune and nodular causes of hyperthyroidism. Whilst thyroid function tests (TFTs) are readily available when a patient first presents to the endocrinologist, in this narrative review we discuss the inclusion of thyroid USS as another first line bedside investigation in thyrotoxicosis.

Given the increasing availability and affordability of high-resolution (including portable) USS devices, there is a de facto inclusion of thyroid sonography as an extension of the bedside examination at many endocrine centres around the globe. Notwithstanding, this is not a universal policy and not all endocrinologists believe that thyroid USS has a role in the diagnosis and management of GD. Indeed, it must be noted that the bedside thyroid USS is not a practice that is as yet endorsed by international guidelines.

The sonographic signature characteristics of GD include a diffuse reduction in echogenicity, linear echogenic inclusions, and increased gland volume, usually with a symmetrical enlargement of the entire gland with consequent displacement of the vascular bundles of the neck laterally and/ or dorsally [2]. However, it is the intense vascularity ('thyroid inferno') on the power Doppler that appears pathognomonic of GD with a positive predictive value of $95 \%$ [3]. Nevertheless, in mild, and treated, GD, the vascularity may be mildly increased or even normal and, conversely, hashitoxicosis may give a markedly increased vascularity [4]. Various techniques do exist to quantify the vascularity using colour Doppler imaging: thyroid blood flow area (TBFA), superior, or inferior, thyroid artery mean peak systolic velocity, and dedicated software calculating thyroid blood flow area [5-8]. In clinical practice, this means that in many cases the diagnosis can be instantly confirmed long before TSH receptor antibody (TRAb) results - which have high sensitivity and specificity for GD [9] - become available. Even in TRAb-positive patients, the co-existence of GD and toxic nodules (or the presence of nodular GD) is of interest, as it may guide the management towards defini- tive treatment. Similarly, it is useful to recognise the co-existence of parathyroid adenomas as this may also influence the management [10]. In a surgical series of 96 patients who underwent thyroidectomy for hyperthyroidism, 13 (13.5\%) were found to have concomitant primary hyperparathyroidism (11 with a parathyroid adenoma and two with hyperplasia) [11]. Another single-centre series reported 21 cases of concomitant GD and primary hyperparathyroidism diagnosed based on clinical, intraoperative parathyroid hormone monitoring and histology criteria [12]. Regardless of the likely selection bias in this single-centre series, the point should be made that the co-existence of these two pathologies is not infrequent and that mild hypercalcaemia in patients with hyperthyroidism should not be assumed to be due to the hyperthyroidism per se.

Furthermore, suspicious and malignant cytology (Bethesda classifications 5 and 6, respectively) is significantly more common in nodules aspirated in patients with GD $(20 \%$ of fine-needle aspirations vs. $7 \%$ in patients without GD) [13]. Indeed, in a systematic review and meta-analysis, which included a total of seven retrospective studies and 2,582 patients with GD, 297 (11.5\%) were found to have thyroid cancer [14]. Thyroid nodules were identified in 968 (37.5\%) patients and were correlated with a fivefold increased risk of thyroid carcinoma vs. those with GD and no nodules on the thyroid USS [14]. Nevertheless, it is worth noting the high study heterogeneity, the retrospective nature and the possible selection bias of the included studies, which enrolled only participants who underwent surgery. Moreover, these studies did not report on ultrasound stratification systems ((e.g. the ATA [15], TIRADS [16], ACR-TIRADS [17] or EU-TIRADS [18]) and, perhaps more importantly, did not report on the percentage of papillary microcarcinomas (microPTC). This was addressed in a retrospective surgical series of 526 patients who underwent thyroidectomy for GD. The above-mentioned study again showed high prevalence of thyroid nodules $(177 / 526,34 \%)$, a significant prevalence of thyroid cancer $(42 / 526,8 \%)$, and a significantly increased risk of thyroid cancer in the presence of a nodule [19]. It also provided information regarding the nature of thyroid cancer; all 42 patients had papillary thyroid carcinoma (PTC), 33 (79\%) had micro-PTC, three $(7 \%)$ had lymph node infiltra- 
tion and 37 (88\%) were deemed to have Stage I disease [19]. During a seven-year follow-up, recurrence was observed in three $(7 \%)$ patients, including one with micro-PTC, and no mortality was seen [19]. On the other hand, some studies reported a higher incidence of aggressive variants of papillary thyroid carcinoma and lymph node metastases $[20,21]$. Pathophysiologically, the increased incidence and aggressiveness of thyroid carcinoma in the context of hyperthyroidism may relate to the stimulatory effect of TRAb on the differentiated thyroid carcinoma cells which retain their TRAb receptors $[22,23]$. Based on the above evidence, an over-diagnosis of papillary micro-carcinomas is a concern when it comes to the widespread application of thyroid sonography. This concern can be ameliorated if the sonography is performed by experienced and formally-accredited sonographers who adhere to international guidelines on the management of thyroid nodules and cancer $[15,17]$.

Moreover, it is worth briefly mentioning the utility of thyroid sonography in the diagnostic work-up of medication-induced thyrotoxicosis. With amiodarone [24], interferon [25] and the newer immune-complex inhibitors [24-26], the distinction is usually between a destructive thyroiditis and hyperthyroidism. Thyroiditis is, more often than not, associated with reduced vascularity and echogenicity on USS and little or no diffuse thyroid enlargement. Conversely, medication-induced hyperthyroidism is more commonly associated with increased vascularity, diffuse thyroid enlargement or a multi-nodular goitre. With the aforementioned medications, along with denileukin diftitox (IL-2 fused to dipthenia toxin), a destructive thyroiditis is the more frequent cause of the thyrotoxicosis, whereas with alemtuzumab [27] Graves' disease, hyperthyroidism appears to be more common, although regarding these latter two medications not a lot of evidence exists in relation to their sonographic signature.

The American Thyroid Association 2016 guidelines on hyperthyroidism state that, if the diagnosis is not apparent clinically and biochemically, then, depending on expertise and resources, measurement of TRAb or radioiodine uptake scintigraphy or measurement of thyroid blood flow on USS can be performed, with preference for scintigraphy when the clinical presentation suggests a toxic adenoma or multi-nodular goitre [10].
However, the reliance on palpation for the identification of thyroid nodules is problematic, with low sensitivity and specificity. For example, in a study of 135 patients with hyperthyroidism, 60 (45\%) were found to have thyroid nodules, of which a third were not felt on palpation [28]. Moreover, considering the aforementioned high incidence of thyroid malignancy in patients who present with hyperthyroidism and thyroid nodules [13], and the delay in performing scintigraphy, the higher costs and the radiation involved with it, it may seem logical for thyroid sonography to be carried out first in all patients. Consequently, scintigraphy can be reserved in a minority of cases, as clinically indicated. Indeed, thyroid sonography appears to be the preferred method of investigation employed by endocrinologists in the modern era. This is reflected in the results of two surveys that examined how endocrinologists manage hyperthyroidism. The first, from France, included 992 patients managed by 263 endocrinologists; thyroid USS was performed in $94 \%$ of the cases (vs. $40 \%$ and $58 \%$ who had scintigraphy and TRAb, respectively) [29]. The second survey, from Italy, included 947 endocrinologists; $92 \%$ reported that they would request a thyroid USS for hyperthyroidism (vs. $25 \%$ for scintigraphy) and, overwhelmingly, chose thyroid sonography in conjunction with TRAb as their preferred diagnostic modalities [30]. Our practice preference in Cyprus is also to perform both thyroid sonography and TRAb, in that sequence, invariably in all patients presenting with hyperthyroidism. It is worth mentioning the better diagnostic performance of TRAb vs. the clinical assessment of hyperthyroidism, as well as the fact that a negative TRAb does not distinguish among other aetiologies and may, on occasion, be seen in very mild GD [1,10,31].

In a recent authoritative review on Graves' disease [32], the utilisation of thyroid sonography was upgraded to a first line investigation, even in patients with clinically suspected hyperthyroidism whose TFTs were not available. The authors highlighted that thyroid USS can allow for the immediate distinction between GD and multi-nodular goitre, which can be achieved on a patient's first presentation and without the need for radiological imaging [32]. TRAb has been recommended as a subsequent investigation tool in all patients for the definitive diagnosis of GD and for aiding management, whereas radioiodine uptake scan 
is considered unnecessary if the patient is TRAb positive [1,32]. These recommendations are congruent with our own practice and the conclusions drawn from this literature review.

\section{Case study}

A 26-year-old female presented to the endocrine clinic with malaise, tremor, restlessness and palpitations for a month. Physical examination revealed resting tachycardia at 100 beats per minute, tremors and a non-tender goitre, but no pathognomonic features of Graves' disease, such as ophthalmopathy, pretibial myxoedema or acropachy. Blood tests (performed prior to the consultation by her general practitioner) showed a free tetraiodothyronine (T4) of 59.9 (reference range, $10.00-19.78) \mathrm{pmol} / \mathrm{L}$ and a supressed TSH at $<0.005(0.25-5.00) \mathrm{mIU} / \mathrm{L}$. Thus, the patient's clinical features and blood tests were consistent with thyrotoxicosis. In the absence of a clinically evident cause, the differential diagnosis included Graves' disease, thyroiditis and nodular disease. A thyroid ultrasound scan performed as an extension of the physical exami-
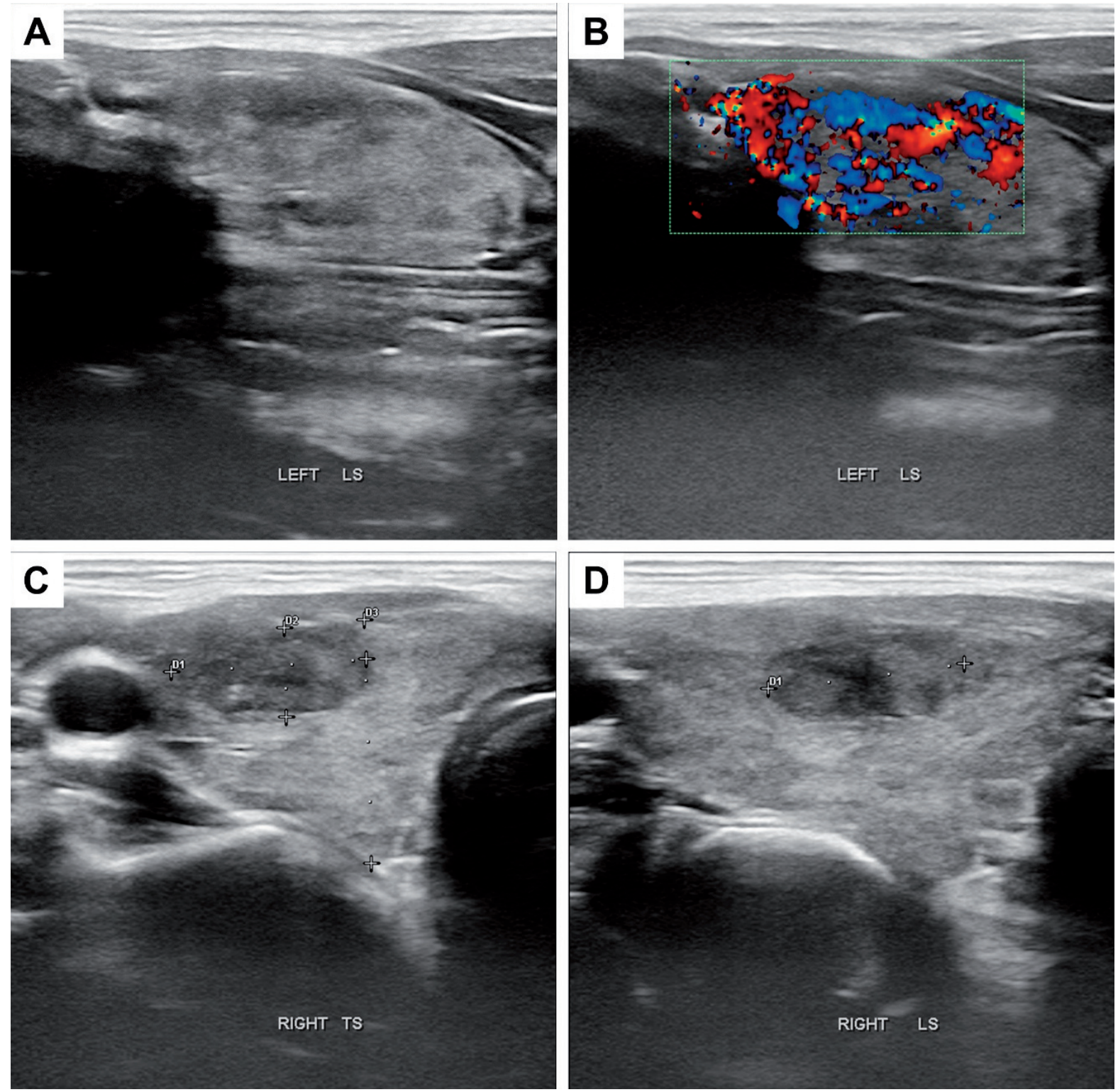

Figure 1. Bedside thyroid ultrasound scan showing an enlarged thyroid with diffuse and marked heterogeneity (A), along with an intensely increased vascularity on power Doppler, indicative of 'thyroid inferno' (B). A thyroid nodule was also visible on the right lobe (C); this measured $16.1 \times 7.3 \times 16.3 \mathrm{~mm}$ and was hypoechoic with irregular borders, subcapsular and in contact, but not clearly infiltrating, the anterior capsule (D) and stratified as a 'high risk' nodule 
nation 'on the spot' revealed an enlarged thyroid with diffuse and marked heterogeneity along with an intensely increased vascularity on the power Doppler, indicative of 'thyroid inferno' (Figure 1). However, a thyroid nodule was also identified in the right lobe; this was hypoechoic with irregular borders, subcapsular and in contact with, but not clearly infiltrating, the anterior thyroid capsule; it was stratified as a 'high risk' nodule as per American Thyroid Association 2015 guidelines on the management of thyroid nodules and cancer [15]. By the end of her first visit to the endocrinologist, a dual diagnosis of Graves' disease and possible thyroid carcinoma was given. She was started on anti-thyroid drugs and counselled that surgery may be required. Subsequently, her TSH receptor antibody (TRAb) titres came back significantly raised, confirming Graves' disease. A radioiodine uptake scan showed reduced uptake in the rightsided nodule region and an ultrasound-guided fine-needle aspiration test showed cytological appearances of papillary thyroid carcinoma (PTC) (Thy 5 or Bethesda 6). A total thyroidectomy with therapeutic central compartment lymph node dissection was successfully performed within six weeks of presentation. Histopathologically, a $15 \mathrm{~mm}$ right-sided PTC was evident with no extrathyroidal extension and no capsular or vascular invasion, with an incidental finding of left-sided $2 \mathrm{~mm}$ and $3 \mathrm{~mm}$ PTC foci. Six out of 17 lymph nodes were also positive for PTC (max. diameter was $4 \mathrm{~mm}$ ). Overall, tumour grading was $\mathrm{pT} 1 \mathrm{~b}(\mathrm{~m}) \mathrm{N} 1 \mathrm{aMOR0}$; stage 1 . Radioactive iodine ablation $(30 \mathrm{mCi})$ was administered three months post-operatively. The patient has since been wellcontrolled on levothyroxine and remains euthyroid to this date (two and a half years after her presentation) with an undetectable thyroglobulin, negative thyroglobulin antibodies and a normal post-thyroidectomy thyroid ultrasound scan, hence no evidence of thyroid cancer recurrence.

\section{Acknowledgements}

\section{Conflict of interest statement}

The authors declare no conflict of interest.

\section{Funding sources}

There are no sources of funding to declare.

\section{References}

1. Bell L, Hunter AL, Kyriacou A, Mukherjee A, Syed AA. Clinical diagnosis of Graves' or non-Graves' hyper- thyroidism compared to TSH receptor antibody test. Endocrine Connections. 2018 Apr;7(4):504-510. https://doi.org/10.1530/ec-18-0082

2. Kharchenko $V$, Kotlyarov $P$, Mogutov $M$, Alexandrov Y, Sencha A, Patrunov Y, Belyaev D, eds. Ultrasound diagnostics of thyroid diseases. First edit. SpringerVerlag Berlin Heidelberg; 2010.

3. Scappaticcio L, Trimboli P, Keller F, Imperiali M, Piccardo A, Giovanella L. Diagnostic testing for Graves' or non Graves' hyperthyroidism: A comparison of two thyrotropin receptor antibody immunoassays with thyroid scintigraphy and ultrasonography. Clinical Endocrinology. 2019 Dec 5;92(2):169-178. https://doi. org/10.1111/cen.14130

4. Baskin $H$, Duick D, Levine R, eds. Thyroid ultrasound and ultrasound-guided FNA. Third edition. Springer; 2013.

5. Kurita S, Sakurai M, Kita Y, Ota T, Ando H, Kaneko S, Takamura T. Measurement of Thyroid Blood Flow Area Is Useful for Diagnosing the Cause of Thyrotoxicosis. Thyroid. 2005 Nov;15(11):1249-1252. https:// doi.org/10.1089/thy.2005.15.1249

6. Uchida T, Takeno K, Goto M, Kanno R, Kubo S, Takahashi S, Azuma K, Sakai K, Fujitani Y, Hirose T, Kawamori R, Watada H. Superior thyroid artery mean peak systolic velocity for the diagnosis of thyrotoxicosis in Japanese patients. Endocrine Journal. 2010;57(5):439-443. https://doi.org/10.1507/endocrj. k09e-263

7. Kumar KH, Pasupuleti V, Jayaraman M, Abhyuday V, Ramasubba Rayudu B, Modi KD. Role of Thyroid Doppler in Differential Diagnosis of Thyrotoxicosis. Endocrine Practice. 2009 10.1111/j.1365-2265 .2007.02832.x1;15(1):6-9. https://doi.org/10.4158/ ep.15.1.6

8. Ota H, Amino N, Morita S, Kobayashi K, Kubota S, Fukata S, Kamiyama N, Miyauchi A. Quantitative measurement of thyroid blood flow for differentiation of painless thyroiditis from Graves? disease. Clinical Endocrinology. 2007 Jul;67(1):41-45. https://doi. org/10.1111/j.1365-2265.2007.02832.x

9. Matthews DC, Syed AA. The role of TSH receptor antibodies in the management of Graves' disease. European Journal of Internal Medicine. 2011 Jun;22(3):213-216. https://doi.org/10.1016/j. ejim.2011.02.006

10. Ross DS, Burch HB, Cooper DS, Greenlee MC, Laurberg $P$, Maia AL, Rivkees SA, Samuels $M$, Sosa JA, Stan MN, Walter MA. 2016 American Thyroid Association Guidelines for Diagnosis and Management of Hyperthyroidism and Other Causes of Thyrotoxicosis. Thyroid. 2016 Oct;26(10):1343-1421. https://doi. org/10.1089/thy. 2016.0229

11. Toursarkissian B, Sloan DA, Schwartz RW. Coexisting hyperthyroidism and primary hyperparathyroidism. Surgery. 1993 Jun;113(6):716-8. PMID 8506532

12. Wei S, Baloch ZW, LiVolsi VA. Parathyroid Adenoma in Patients with Graves' Disease: a Report of 21 Cases. Endocrine Pathology. 2014 Dec 13;26(1):71-74. https://doi.org/10.1007/s12022-014-9349-0

13. Hadjisavva IS, Dina R, Talias MA, Economides PA. Prevalence of Cancer in Patients with Thyroid Nod- 
ules in the Island of Cyprus: Predictive Value of Ultrasound Features and Thyroid Autoimmune Status. European Thyroid Journal. 2015;4(2):123-128. https:// doi.org/10.1159/000430438

14. Papanastasiou A, Sapalidis K, Goulis DG, Michalopoulos N, Mareti E, Mantalovas S, Kesisoglou I. Thyroid nodules as a risk factor for thyroid cancer in patients with Graves' disease: A systematic review and meta analysis of observational studies in surgically treated patients. Clinical Endocrinology. 2019 Aug 13;91(4):571-577. https://doi.org/10.1111/ cen.14069

15. Haugen BR, Alexander EK, Bible KC, Doherty GM, Mandel SJ, Nikiforov YE, Pacini F, Randolph GW, Sawka AM, Schlumberger M, Schuff KG, Sherman SI, Sosa JA, Steward DL, Tuttle RM, Wartofsky L. 2015 American Thyroid Association Management Guidelines for Adult Patients with Thyroid Nodules and Differentiated Thyroid Cancer: The American Thyroid Association Guidelines Task Force on Thyroid Nodules and Differentiated Thyroid Cancer. Thyroid. 2016 Jan;26(1):1133. https://doi.org/10.1089/thy. 2015.0020

16. Grant EG, Tessler FN, Hoang JK, Langer JE, Beland MD, Berland LL, Cronan JJ, Desser TS, Frates MC, Hamper UM, Middleton WD, Reading CC, Scoutt LM, Stavros AT, Teefey SA. Thyroid Ultrasound Reporting Lexicon: White Paper of the ACR Thyroid Imaging, Reporting and Data System (TIRADS) Committee. Journal of the American College of Radiology. 2015 Dec;12(12):1272-1279. https://doi.org/10.1016/j. jacr.2015.07.011

17. Tessler FN, Middleton WD, Grant EG, Hoang JK, Berland LL, Teefey SA, Cronan JJ, Beland MD, Desser TS, Frates MC, Hammers LW, Hamper UM, Langer JE, Reading CC, Scoutt LM, Stavros AT. ACR Thyroid Imaging, Reporting and Data System (TIRADS): White Paper of the ACR TI-RADS Committee. Journal of the American College of Radiology. 2017 May;14(5):587-595. https://doi.org/10.1016/j. jacr.2017.01.046

18. Russ G, Bonnema S, Erdogan M, Durante C, Ngu R, Leenhardt L. European Thyroid Association Guidelines for Ultrasound Malignancy Risk Stratification of Thyroid Nodules in Adults: The EU-TIRADS. European Thyroid Journal. 2017;6(5):225-237. https://doi. org/10.1159/000478927

19. Tam AA, Kaya C, Kılıç FBM, Ersoy R, Çakır B. Thyroid nodules and thyroid cancer in Graves' disease. Arquivos Brasileiros de Endocrinologia \& Metabologia. 2014 Dec;58(9):933-938. https://doi. org/10.1590/0004-2730000003569

20. Chen $Y$, Lin C, Chang $Y$, Cheng FT, Peng $C$, Sung F, Cheng Y, Kao C. Cancer Risk in Patients with Graves' Disease: A Nationwide Cohort Study. Thyroid. 2013 Jul;23(7):879-884. https://doi.org/10.1089/ thy. 2012.0568

21. Boutzios G, Vasileiadis I, Zapanti E, Charitoudis $G$, Karakostas $E$, leromonachou $P$, Karatzas $T$. Higher Incidence of Tall Cell Variant of Papillary Thyroid Carcinoma in Graves' Disease. Thyroid.
2014 Feb;24(2):347-354. https://doi.org/10.1089/ thy. 2013.0133

22. Preece J, Grodski S, Yeung M, Bailey M, Serpell J. Thyrotoxicosis does not protect against incidental papillary thyroid cancer. Surgery. 2014 Nov;156(5):11531156. https://doi.org/10.1016/j.surg.2014.04.025

23. Fu $H$, Cheng $L$, Jin $Y$, Chen $L$. Thyrotoxicosis with concomitant thyroid cancer. Endocrine-Related Cancer. 2019 Jul;26(7):R395-R413. https://doi.org/10.1530/ erc-19-0129

24. Eaton SEM, Euinton HA, Newman CM, Weetman AP, Bennet WM. Clinical experience of amiodarone-induced thyrotoxicosis over a 3-year period: role of colour-flow Doppler sonography*. Clinical Endocrinology. 2002 Jan;56(1):33-38. https://doi.org/10.1046/ j.0300-0664.2001.01457.x

25. Kyriacou A, McLaughlin J, Syed AA. Thyroid disorders and gastrointestinal and liver dysfunction: A state of the art review. European Journal of Internal Medicine. 2015 Oct;26(8):563-571. https://doi. org/10.1016/j.ejim.2015.07.017

26. Iyer PC, Cabanillas ME, Waguespack SG, Hu MI, Thosani S, Lavis VR, Busaidy NL, Subudhi SK, Diab A, Dadu R. Immune-Related Thyroiditis with Immune Checkpoint Inhibitors. Thyroid. 2018 Oct;28(10):12431251. https://doi.org/10.1089/thy.2018.0116

27. Pariani N, Willis M, Muller I, Healy $S$, Nasser T, McGowan A, Lyons G, Jones J, Chatterjee K, Dayan C, Robertson N, Coles A, Moran C. Alemtuzumab-Induced Thyroid Dysfunction Exhibits Distinctive Clinical and Immunological Features. The Journal of Clinical Endocrinology \& Metabolism. 2018 Jun 6;103(8):3010-3018. https://doi.org/10.1210/jc.201800359

28. Varadhan L, Varughese G, Sankaranarayanan S. Hyperthyroidism and Graves' disease: Is an ultrasound examination needed?. Indian Journal of Endocrinology and Metabolism. 2016;20(6):866. https:// doi.org/10.4103/2230-8210.192899

29. Goichot B, Bouée S, Castello-Bridoux C, Caron P. Survey of Clinical Practice Patterns in the Management of 992 Hyperthyroid Patients in France. European Thyroid Journal. 2017;6(3):152-159. https://doi. org/10.1159/000453260

30. Negro R, Attanasio R, Grimaldi F, Guglielmi R, Papini E. A 2015 Italian Survey of Clinical Practice Patterns in the Management of Graves' Disease: Comparison with European and North American Surveys. European Thyroid Journal. 2016;5(2):112-119. https://doi. org/10.1159/000444482

31. Matthews DC, Syed AA. The role of TSH receptor antibodies in the management of Graves' disease. European Journal of Internal Medicine. 2011 Jun;22(3):213-216. https://doi.org/10.1016/j. ejim.2011.02.006

32. Davies TF, Andersen S, Latif R, Nagayama Y, Barbesino G, Brito M, Eckstein AK, Stagnaro-Green A, Kahaly GJ. Graves' disease. Nature Reviews Disease Primers. 2020 Jul 2;6(1). https://doi.org/10.1038/s41572020-0184-y 